\title{
Overview of Cytological Dynamics of Periodontal Ligament Inflammatory Lesions
}

Review Article

\author{
Matsuda $\mathrm{S}^{1,2}$, Nakano $\mathrm{K}^{3,4}$, Tsujigiwa $\mathrm{H}^{5}$, Takabatake $\mathrm{K}^{3}$, Okafuji $\mathrm{N}^{1}$, Shoumura $\mathrm{M}^{2}$, Osuga $\mathrm{N}^{2}$, Nagatsuka $\mathrm{H}^{3}$, Kawakami $\mathrm{T}^{1^{*}}$ \\ ${ }^{1}$ Department of Hard Tissue Research, Matsumoto Dental University Graduate School of Oral Medicine, Shiojiri, Japan. \\ ${ }^{2}$ Department of Pediatric Dentistry, Matsumoto Dental University School of Dentistry, Shiojiri, Japan. \\ ${ }^{3}$ Department of Oral Pathology and Medicine, Graduate School of Medicine, Dentistry and Pharmaceutical Sciences, Okayama University, Okayama, \\ Japan. \\ ${ }^{4}$ Department of Oral Pathology, Matsumoto Dental University School of Dentistry, Shiojiri, Japan. \\ ${ }^{5}$ Department of Life Science, Faculty of Science, Okayama University of Science, Okayama, Japan.
}

\section{Abstract}

Cyto-pathological features of the periodontal ligament tissue inflammatory lesions have somehow been carried out but detailed cellular dynamics remain unclear. Therefore, in this review, we overviewed mainly our recent experimental model studies. That is performed using using ordinary ddY mice and BMP bone marrow transplanted mouse model. Regaring the experimental apical inflammatory periodontitis, at four weeks, micro-CT confirmed the presence of a radiolucent image at the apex of the tooth, which was then removed for histological examination. The results showed granulation tissue with fibrosis gradually formed at the periphery of an abscess.

Next, if perforation were large, granulation tissue would grow to form periodontal polyp. Results of micro-CT revealed alveolar bone resorption and widening of periodontal ligament. Histopathological examination showed proliferation of fibroblasts with some round cells and blood vessels in the granulation tissue.

Double immunofluorescent staining of GFP and Runx2 revealed that both proteins were expressed in spindle-shaped cells. Double immunofluorescent staining of GFP and CD31 revealed that both proteins were expressed in vascular endothelial cells in morphologically distinct vessels. The results suggest that fibroblasts, periodontal ligament fibroblasts and blood vessels in granulation tissue were derived from transplanted-bone marrow cells. Thus, essential growth of granulation tissue in periodontal polyp was caused by the migration of undifferentiated mesenchymal cells derived from bone marrow, which differentiated into fibroblasts and later on differentiated into other cells in response to injury.

Keywords: Chronic Apical Periodontitis; Alveolar Abscess; Chronic Proliferelative Periodontal Inflammatory Lesion; Periodontal Polyp; Granulation Tissue; Periodontal Ligament; Cell Differentiation; Cell Migration; Undifferentiated Mesenchymal Cells; Bone Marrow.

\section{Introduction}

Inflammatory lesion, such as apical periodontitis, is a disease characterized by tissue destruction at the root apex of tooth said to be a host immune response due to bacterial infection [1-4]. The involvement of cytokines produced during inflammation has been mentioned in studies involving bone lesions [5, 6]. Various factors inducing inflammatory response, osteoclast activation, etc., in bone destruction have been reported. However, extensive cellular dynamics, growth, infiltration and the mechanism of formation are still unclear.

Therefore, we attempted to establish a convenient experiment in animal model using ddY mouse, which can be verified. The method also focused on the histopathological findings of chronic inflammatory apical lesion including the immunohistochemical findings. Cytological dynamics of the periodontal ligament tissue inflammatory lesions have somehow been carried out but detailed

\footnotetext{
*Corresponding Author:

Toshiyuki Kawakami,

Professor, Hard Tissue Pathology Unit, Department of Hard Tissue Research, Matsumoto Dental University Graduate School of Oral Medicine, Shiojiri, Japan.

Tel: +81-263-51-2035

E-mail: kawakami@po.mdu.ac.jp
}

Received: August 08, 2016

Accepted: September 07, 2016

Published: September 08, 2016

Citation: Matsuda S, Nakano K, Tsujigiwa H, Takabatake K, Kawakami T, et al., (2016) Overview of Cytological Dynamics of Periodontal Ligament Inflammatory Lesions. Int J Dentistry Oral Sci. S9:001, 1-7. doi: http://dx.doi.org/10.19070/2377-8075-SI09001

Copyright: Kawakami $\mathbf{T}^{\circ}$ 2016. This is an open-access article distributed under the terms of the Creative Commons Attribution License, which permits unrestricted use, distribution and reproduction in any medium, provided the original author and source are credited. 
cellular dynamics remain unclear. Therefore, in this review, we introduce mainly our recent experimental mouse model studies.

\section{Chronic Apical Periodontitis (Alveolar Abscess)}

Periapical lesion is a disease caused by bacterial infection characterized by destruction of the apical periodontal tissues by osteoclasts coupled with expanding inflammation. Cytokines produced during inflammation are said to be involved in the pathogenesis and the role of local inflammation has been associated with bone resorption [1-4]. An inflammatory cytokine called Interleukin-1 is involved in tissue breakdown and expansion of inflammatory response. It enhances the production of collagenase and prostaglandins indirectly via cytokine production and it is also involve in inflammatory reaction and bone destruction. It has also been suggested that cytokines produced by the inflammatory tissue at the apex of the tooth induce osteoclasts to act on osteoblast lineage to inhibit bone formation $[5,6]$. We thought to verify this by establishing a simple animal experiment clarifying the pathogenesis of apical periodontitis.

Although other authors reported the induction of periapical lesion by allowing pulp exposure of rat molars $[7,8]$, studies on laboratory animals using micro-CT over time are very few [5]. In conventional micro-CT, the $\mathrm{x}$-ray tube and sensor are fixed therefore the image is difficult to aim since the tail and head would contact the tube while rotating in vivo, which is essential in sample preparation. However, Arai et at., [9-12] developed a micro-CT with a stage holding small animals such as laboratory mice stable. In this way the sensor and tube can be rotated thus, it is possible to obtain a three-dimensional image of 100 million pixels in a short recording time of 17 seconds. Thus, we used micro-CT.

In our recent animal examination results are as followed [13]. Some day male mice were used in the experiment. Under general anesthesia tincture of iodine was used to disinfect the left maxillary first molar. Using one-half round bur (Maillefer, Ballaiques) and drill, the coronal portion of the pulp was exposed and allowed to penetrate until the apex. The opening was confirmed using a size 20 file. After pulp exposure, post-operative micro-CT was done to confirm the treatment. After confirmed, the regional specimens were then subjected to histopathological examinations. Furthermore, immunohistochemistry (IHC) was performed.

In the examination, micro-CT observation shows normal pulp cavity of the maxillary first molar specifically the mesial root being the subject of the experiment. The root canal of the tooth is normal having an approximately equal width of dentin on both sides of the root canal. The periodontal membrane is relatively wide but its width is uniform; apical radiolucency suggestive of inflammatory apical lesion was not observed. After pulp exposure of the coronal portion and penetration until the root apex of the first molar, size 20 file was used to confirm the patency of root canal in micro-CT image; the opening toward the center of pulp cavity could be clearly observed. Loss of dentin and its immediate enamel crown is evident. The width of the lumen of root canal lumen became irregular clearly indicating the effect of the instrument. Two weeks later, micro-CT revealed a small radiolucency at the root apex, which increased in size after 4 weeks. The external part is opened from the central part of the crown slightly induced by caries. The radiolucent image appeared at the apex of the mesial root. The radiolucent image continued to grow in the apical portion. Inside the radiolucency, a portion of the root apex could be seen protruding. The radiolucency was relatively uniform from micro-CT image. In addition, radiopaque line at the border of the radiolucent image was detected (Figure $1)$.

Histopathological examination of the tissue removed at the apex of the maxillary first molar 4 weeks after surgery, revealed the presence of three abscesses surrounded by granulation tissue (Figure 2-a,b). At high magnification, the center is filled with neutrophils, lipids and pus. Furthermore, numerous macrophages in the same area where pus is growing slowly were noted. The abscess is surrounded by a young granulation tissue, which gradually became fibrotic. Macrophages and fibroblasts were also observed in the same area (Figure 2-c). The macrophages are large with light and granular cytoplasm hence, are also called foam cells. IHC with CD31 showed positive reaction in the center of the lesion although capillaries positive to CD31 were hardly seen. Cells positive to CD 31 were confined in granulation tissue in the area of fibrosis relatively around the abscess (Figure 2-d). Kimberly et al., [7] showed in their study that exposure of the pulp in rat molars would lead to necrosis of the entire pulp and granulation tissue formation six days post-operatively and necrotic pulp tissue remains until 5 weeks which then proceeds to the formation of granulation tissue at the apex of the tooth. In this study, it is clear that the cavity opened towards the pulp cavity of the crown and radiolucency emerged at the mesial root apex 4 weeks later using micro-CT. Furthermore, when the maxillary first molar was removed for histopathological examination, an abscess bounded mostly by immature and fibrotic granulation tissue was

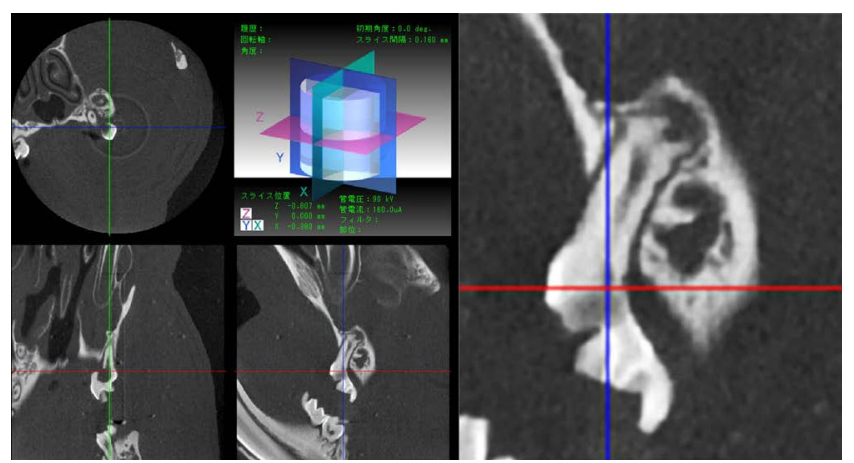

Figure 1. Micro-CT image of one month specimen. Right is the enlarged image. Radiolucent image showing formed lesion continuously to the root apex. 


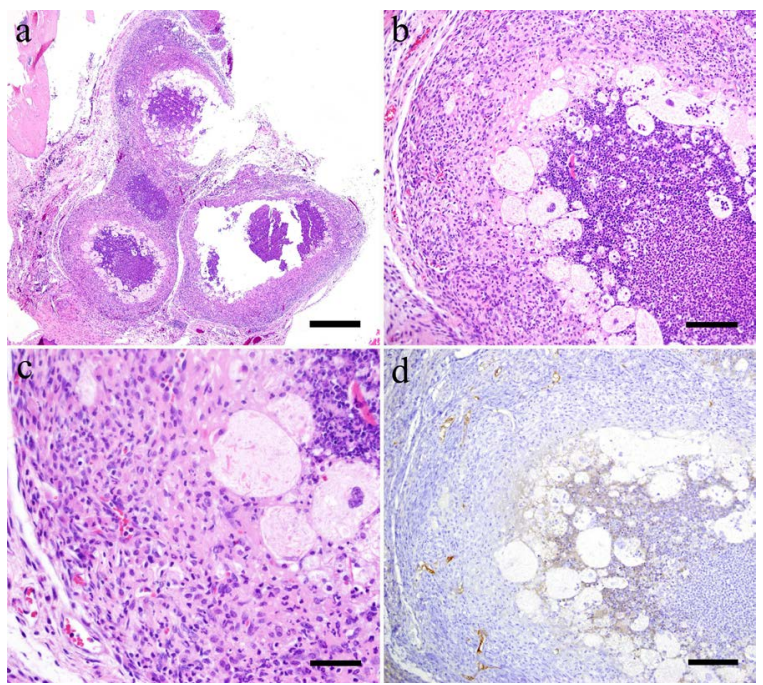

Figure 2. Histopathological ptotograph. a. Abscess formed within the granulation tissue (scale bar: $0.5 \mathrm{~mm}$ ); $\mathrm{b}$ and c. A high power views showing many macrophages at the periphery of the abscess surrounded by granulation tissue (scale bar: b: $100 \mu \mathrm{m}$; b: $50 \mu \mathrm{m}$ ); c. CD-31 positive cells are present at the periphery of the granulation tissue (scale bar: $100 \mu \mathrm{m})$.

observed. The periapical lesion fabricated experimentally could be considered equivalent to the clinical chronic apical periodontitis.

Furthermore, Tsujigiwa et al., [14] established an experiment on GFP bone marrow transplanted mice locating the cells that migrated to various tissues using mesenchymal cells in the bone marrow. The study also revealed that the mesenchymal stem cells differentiated into a variety of cell types in their local destination [15-18]. Therefore, the present experiment system together with GFP bone marrow transplant mice established by Tsujigiwa et al., [14] would contribute greatly to the understanding of the mechanism in the formation of chronic apical periodontitis.

\section{Chronic Proliferelative Periodontal Inflammatory lesion (Periodontal Polyp)}

In routine dental clinical practice, perforation of floor of the dental pulp is a common occurrence during root canal treatment for dental caries. These problems tend to be more when treating deciduous teeth in clinical pediatric dentistry. The consequence of a large perforation is chronic inflammatory hyperplasia [19]. Granulation tissue grows in the periodontal ligament from the perforated dentin causing periodontal polyp, which may also grow into the pulp cavity. Experimental histopathological studies have been done on periodontal polyp for a long time including histological analysis and treatment [20-23]. However, the origin of the cells has not been elucidated. Consequently, using an experimental system on GFP mouse bone marrow transplantation, this study revealed that the cells were derived from mesenchymal cells of the bone marrow. Our research group has been conducting studies on migration and differentiation of cells in many sites in the oral cavity and teeth by using an experimental system of GFP bone marrow transplanted mice. Tsujigiwa et al., [24] mentioned that transplanted-bone marrow-derived cells migrated to the pulp and then differentiated into pulp tissue cells in GFP mice. Muraoka et al., [25] showed that bone marrow-derived cells migrated to the periodontal tissue and then differentiated into periodontal ligament cells like macrophages and osteoclasts. Moreover, Tomida et al., [15] described the pluripotency of bone marrow-derived cells, which migrated to periodontal tissue after orthodontic mechanical stress load application. Likewise, Kaneko et al., [18] cited the differentiation of cells into cellular components of the periodontal tissue.

In our recent animal study, the cellular components of the granulation tissue to induce chronic inflammation at the furcation in GFP mouse were carried out [19]. That is, also some GFP transplanted mice were used. Under general anesthesia, the mouse was fixed on the plate and hole was drilled on the crown of maxillary left first molar using $1 / 2$ round bur to create a perforation of floor of the dental pulp. The animals were sacrificed at 2 weeks, 1 month, 3 months and 6 months. The tissues were then removed and photographed using micro-CT. At 2 weeks, there was a slight resorption of alveolar bone and expansion of the width of periodontal ligament underneath the perforation (Figure 3-a). At 1 month, the expansion of the width of periodontal ligament became wider and at 3 months, resorption of the alveolar bone was more apparent and the crown of the tooth has completely collapsed (Figure 3-e). At 6 months, widening of the periodontal ligament from furcation to the apex of the root was evident.

At 2 weeks, slight neutrophil infiltration immediately below the perforation was spotted in which a portion was in contact with the dentin wall. The proliferating cells were similar in morphology to the fibroblasts, which are short and spindle in shape with relatively round nucleus. There was an irregularity in the arrangement. Capillaries were present to a certain degree in some places in the granulation tissue. Polygonal epithelial cells with distinct intercellular bridges covered the outermost layer of the granulation tissue. Multinucleated giant cells were observed in some Howship's lacuna (Figure 3-b,c). The cells showed positive red staining with TRAP. At 1 month, the fibroblasts with round nucleus seen at 2 weeks were also spotted but not in the main part of the granulation tissue. The granulation tissue directly underneath the perforation became bigger and grew inside the pulp chamber; the outermost layer is covered with stratified squamous epithelium. The basal layer of the epithelium showed some sort of activity. Proliferation of capillaries increased compared to 2 weeks and most of them were found just beneath 


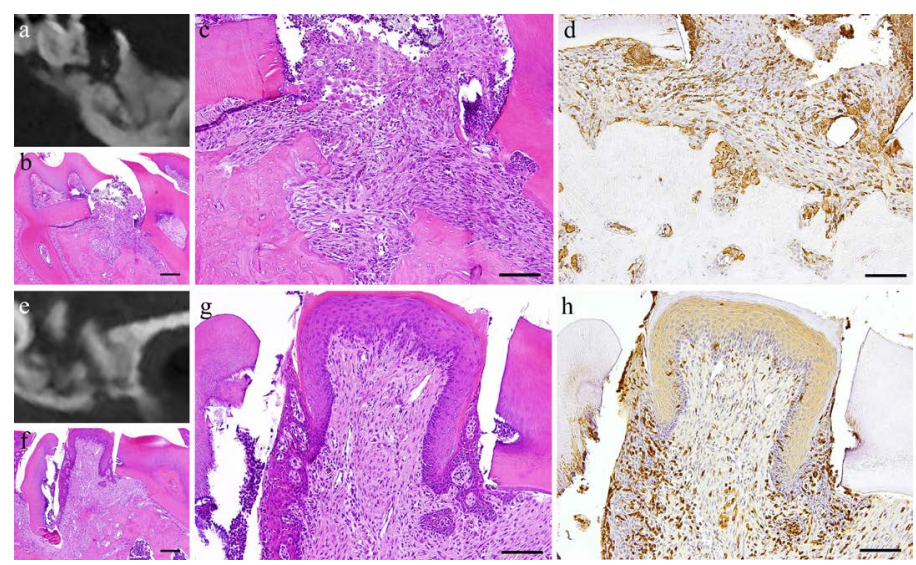

Figure 3. (1) 2-week-specimen. a: Micro CT image; b: Histopathological view of thesame part of a, Scale bar=200 $\mu \mathrm{m}$; c: Enlarged view of b, Scale bar $=100 \mu \mathrm{m}$; $d$ : IHC for GFP, Scale bar $=100 \mu \mathrm{m}$.

(2) 3-month-specimen. e: m_CT image; f: Histopathological view of the same part of a, Scale bar=200 $\mu \mathrm{m}$; g: Enlarged view of $h$, Scale bar $=100 \mu \mathrm{m}$; : IHC for GFP, Scale bar $=100 \mu \mathrm{m}$.

the epithelium. At 3 months, the nucleus of fibroblasts in the granulation tissue became flat. Proliferation of collagen fibers was also evident. Likewise, the stratified squamous epithelial lining became thicker. Capillaries in the granulation tissue also increased at its peak (Figure 3-f, g). At 6 months, there were continuous growth in the granulation tissue, increased collagen fibers, more fibroblasts with more conspicuous flat nucleus and scattered lymphocytes. However, capillaries have slightly decreased.

IHC shows that, at 2 weeks, long and spindle in shape cells with spindle shape nucleus as well as vascular endothelial cells were positive to GFP. In addition, multinucleated giant cells in the area of alveolar bone resorption were also positive to GFP (Figure 3-d). At 1 month, many GFP-positive cells were present in the periodontal polyp underneath the perforation. At 3 months, the spindle-shaped cells and polygonal cells were also positive to GFP. Furthermore, the epithelial layer was negative to GFP and the cells exhibited regular morphology (Figure 3-h). However, some GFPpositive cells, having irregular cytoplasm in shape, were observed. GFP-positive reaction was continuously detected at 6 months. GFP-positive cells were detected as early as 2 weeks and then an increase in expression was noted at 1 month. Nevertheless, no further increase was observed thereafter. The cells inside the capillary were not clear but their expression of CD31 made them discernible. The most number of capillaries was observed at 3 months.

Accidental perforation of floor of the dental pulp is contingent during endodontic treatment. Various conventional dental materials have been used to seal the perforation but not all prognoses were certainly good. Oka et al., [20] used rat to observe the response of periodontal tissue at the furcation associated with medullary pulpal perforation. In a similar study, Imaizumi et al., [21] discussed the enlargement of inflammatory lesion at the furcation. Other animal studies presented the formation of inflammatory lesion as a consequence of perforation of the floor of the pulp chamber. Kudo et al., [22] and Ishida et al., [23] as well as other numerous researches showed the histopathological analysis and treatment following perforation at the furcation area. A detailed histopathological examination by Nakamura et al., [27] showed the continuous growth of granulation tissue in the periodontal ligament. Moreover, to separate and distinguish the periodontal polyp from the growth of the pulp tissue is difficult. Although previous studies have been made, the focus was just on histopathological examination and the origin of the cellular components of the granulation tissue at the furcation was not mentioned.

Recently, the role of undifferentiated mesenchymal cells derived from bone marrow has been widely studied to play a role in periodontal tissue, bone and in other organs. By using bone defect, cementoblast and fibroblast-like cells were produced in the periodontal ligament and alveolar bone by undifferentiated mesenchymal cells from the bone marrow [28-30]. Since GFP transgenic mice expressed the protein, all cells that constitute the tissue and even those that differentiated into any cell from transplanted bone marrow-derived cells can be traced because they carry the protein $[31,32]$. Thus, the study used an experimental system using GFP mice and induced perforation of floor of the dental pulp of maxillary left first molar. With the development of the lesion at the furcation, the origin of the cellular component responsible for the growth of the periodontal polyp was clarified.

Results showed that at 2 weeks, inflammatory cells such as neutrophils were present in response to the physiological mechanism of the periodontal tissue. This was followed by the increase in the fiber component of the granulation tissue as the number of inflammatory cells decreased. Experimental perforation of floor of the dental pulp in mice caused a slight initial suppurative inflammation leading to granulation tissue growth due to gradual chronic inflammation. Similar results were observed from previous experiment [29].

Cells having spindle-shaped nucleus, blood vessels and multinucleated giant cells in areas of bone resorption immediately below the perforation were positive to GFP. Moreover, GFPpositive cells were also spotted to have infiltrated the epithelium, although there were no positive cells in the epithelium. Therefore, the cells are considered to be dendritic cells. Thus, it would also be reasonable to consider that the cells were derived from mesenchymal cells of the bone marrow. In order to identify the GFP-positive cells, double FIHC staining for each marker was performed. 


\section{Cell Differentiation of Bone Marrow-derived} Cells into PDL-Specific Cells in Periodontal Inflammatory Lesions

In our above animal study, immunofluorescence double staining of GFP and S100A4 from 2 weeks to 6 months showed GFPpositive cells with green fluorescence in long spindle-shaped cells with spindle-shape nucleus (Figure 4-a) and S100A4 was also detected in cell outline in red fluorescence (Figure 4-b). Superimposition of both proteins was confirmed in areas where orange stain was found (Figure 4-c). Superimposition in the nucleus was also captured by the orange color with blue fluorescence at periphery of the nucleus. Immunofluorescence double staining of GFP and Runx 2 showed both spindle cells with round nucleus and elongated cells in the granulation tissue were positive to GFP emitting green fluorescence (Figure 5-a) and Runx2 was also detected in the outline of the cells in red fluorescence (Figure $5-b)$. During superimposition, a match between the two proteins was observed indicated by the orange stain (Figure 5-c). Further superimposition with DAPI showing blue fluorescence in the nucleus showed orange stain at the periphery of the nucleus.

Therefore, in our examination results, Runx2-positive and GFP-positive cells that developed an orange stain indicated superimposition of the two proteins. A significant increase in superimposition was observed from 2 weeks to 1 month then decreased at 3 months. The superimposition at 6 months indicated the reaction of both Runx2-positive cells and GFP-positive cells. However, there were many cells only stained individually by GFP. Both GFP and CD31 were clearly expressed by cells in the blood vessels. GFP expression in the cytoplasm of vascular endothelial cells was marked by green fluorescence (Figure 6-a) and CD31 positive reaction was marked by red fluorescence (Figure 6-b). Superimposition of the two proteins was least at 2 weeks (Figure 6-c). At 1 month, an increase in both CD31 and GFP-positive cells was observed.

Muraoka et al., [25] and Kaneko et al., [26] strongly suggested that
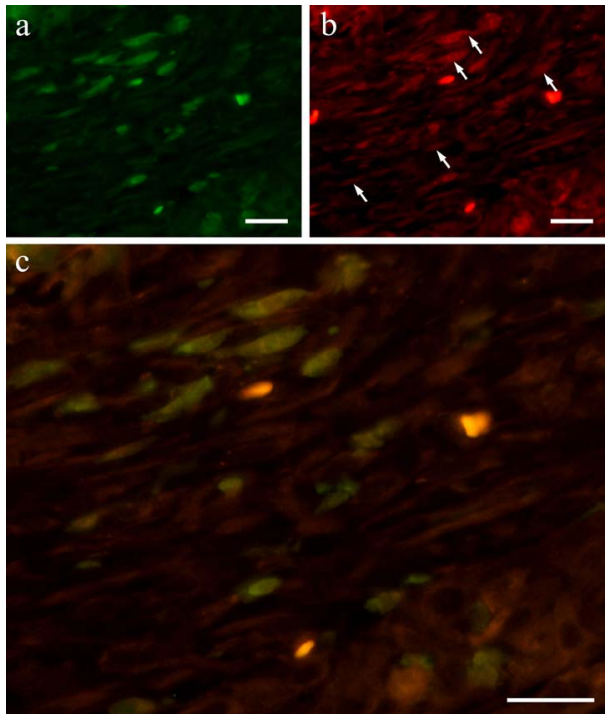

Figure 4. FIHC images of periodontal polyp (arrows indicate the double positive cells) (a: GFP; b: S100A4; c: merged image of GFP and S100A4; 2 week specimen; scale bar $=20 \mu \mathrm{m})$.
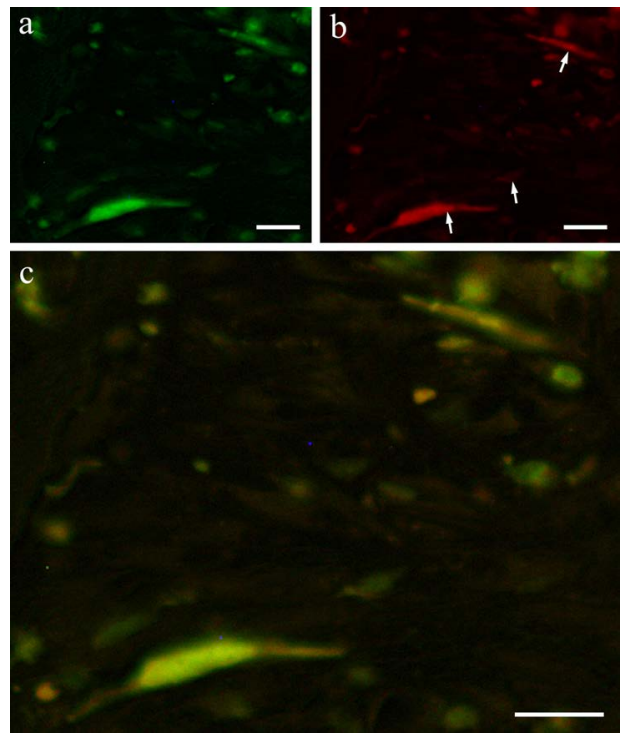

Figure 5. FIHC images of periodontal polyp(arrows indicate the double positive cells) (a: GFP; b: Runx2; c: merged image of GFP and Runx2; 2 week specimen; scale bar $=20 \mu \mathrm{m})$. 

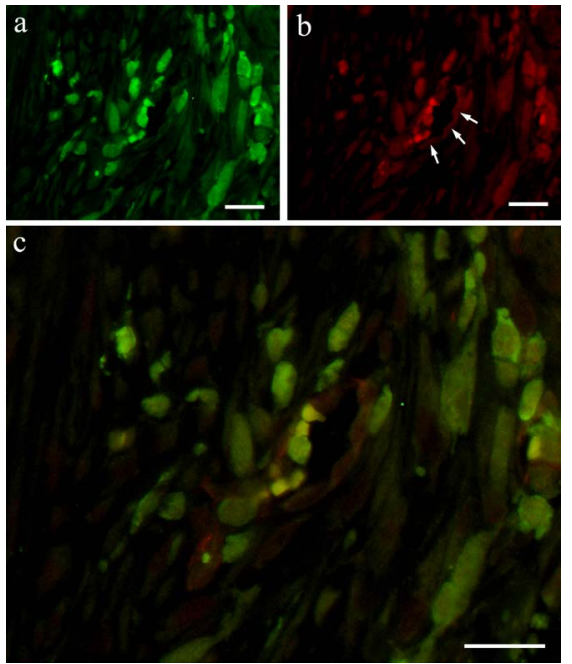

Figure 6. FIHC images of periodontal polyp(arrows indicate the double positive cells) (a: GFP; b: CD31; c: merged image of GFP and CD31; 2 week specimen; scale bar $=20 \mu \mathrm{m})$.

fibroblasts originated from the migration and differentiation of bone marrow cells to the periodontal ligament when experimentally induced. In order to identify the fibroblasts, S100A4 and Runx2 were used since there is no perfect marker for fibroblasts [33-35]. The expression of S100A4 in periodontal ligament was reported to be blocking the calcification in periodontal ligament [36]. However, Saito et al., [37] and Watanabe et al., [38] mentioned that periodontal ligament fibroblast is different from the ordinary fibroblast in homeostasis where in Runx2 is weakly expressed. Many spindle cells with spindle nucleus were positive to S100A4. These cells were also positive to GFP, which means that these cells were undifferentiated mesenchymal cells that migrated and differentiated into fibroblasts. Runx 2 was examined and a number of spindle shaped cells with spindle shaped nuclei were both positive to GFP and Runx2. Thus, these cells were derived from transplanted-bone marrow cells, which later on differentiated into local periodontal ligament fibroblasts. Moreover, the fibroblastlike cells that were positive to both S100A4 and Runx2 were derived from the bone marrow.

Sakai et al., [39] observed the reaction of granulation tissue from foreign substances in the body and a portion of the fibroblasts and capillary endothelial cells were revealed to be bone marrow-derived mesenchymal cells. Immunostaining with CD31, a marker of endothelial cells showed positive reaction in vascular endothelial cells, which also showed positive reaction to GFP. Therefore, some of the capillaries were obtained from the migration and differentiation of undifferentiated mesenchymal cells from the bone marrow cells. Similar observation was also mentioned by Sakai et al., [39] in which chronic inflammation and angiogenesis progressed constantly not only due to the local endothelial cell proliferation but also probably due to the continuous the migration of mesenchymal cells from the bone marrow. The data shown by this review is our experiment result $[13,40]$.

From the above results and overall consideration, fibroblasts, periodontal ligament fibroblasts and blood vessels are essential in the growth of periodontal polyp due to perforation on the floor of the pulp chamber. Furthermore, the osteoclasts observed on the surface of the alveolar bone beneath the perforation originated from transplanted bone marrow cells.

\section{Conclusion}

In the review, we overviewed mainly our recent experimental model studies. Regarding the experimentally induced chronic apical periodontitis (alveolar abscess), the granulation tissue with fibrosis gradually formed at the periphery of an abscess. Next, experimentally induced periodontal polyp, proliferation of fibroblasts with some round cells and blood vessels in the granulation tissue were evaluated. Double immunofluorescent staining results suggest that fibroblasts, periodontal ligament fibroblasts and blood vessels in granulation tissue were derived from transplanted-bone marrow cells. Thus, essential growth of granulation tissue in periodontal polyp was caused by the migration of undifferentiated mesenchymal cells derived from bone marrow, which differentiated into fibroblasts and later on differentiated into other cells in response to injury.

\section{Acknowledgments}

This study was supported in part by Grant-in-Aid for Scientific Research (C) \#23592951, \#23593075, \#25463204, \#26463104 and \#26463031 from the Japan Society for the Promotion of Science. This study was also supported in part by 2016 Futokukai Grant-in-Aid for Scientific Research.

\section{References}

[1]. Dinarello CA (1989) Interleukin-1 and its biologically related Cytokines. Adv Immunol. 44: 153-205.

[2]. Lim GC, Torabinejad M, Kettering J, Linkhardt T, Finkleman RD (1994) Interleukin $1 \beta$ in sympotomatic and asympomatic human periradicular lesions. J Endod. 20(5): 225-227.

[3]. Stashenko P, Obernesser MS and Dewhirst FE (1989) Effect on immune cytokines on bone. Immunol Invest. 18: 239-249.

[4]. Yamada K (1996) Immunohistochemical study on distribution and response of nerve fibers in the healing process of Rat periapilesions.J Fukuoka Dent Coll 23: 387-406.

[5]. Oseko F, Yamamoto T, Yuan LT, Nakanishi A, Kita M, et al., (2006) Threedimensional quantitation of periaptical lesions in mice by micro-computed tomography and immunologic examination. J Conserv Dent 49: 303-308.

[6]. Mori H, Sekine G, Yoshida T and Sekine I (2007) Canges of bone matrix proteins during healing process of periapical lesion. J Conserv Dent 20:582- 
595

[7]. Kimberly CL and Byers MR (1988) Inflamation of rat molar pulp and periodontium causes increased calcitionin gene-rerated peptide and axonal sprouting. Anat Rec. 222: 289-300.

[8]. Krxseicki RF, Fleming WE, Winterrowd GE, Hatfield CA, Sanders MEm et al., (1991) T lymphocyte adhesions to human synovial fibroblasts. Role of cytokines and the interaction between intercellular adhesion molecule 1 and CD11a/CD18. Arthritis Rheum. 34(10): 1245-1253.

[9]. Arai Y, Honda K, Iwai K and Shinoda K (2001) Practical Model "3DX" of limited cone-beam X-ray CT for Dental use. Car 01 Computer assisted radiology and surgery. Elsevier Amsterdam. 671-675.

[10]. Arai Y, Yamada A, Ninomiya T, KatoT and Masuda Y (2005) New micro computed tomography (R_mCT) developed for in vivo animal experiment. Oral Radiorogy 21: 14-18.

[11]. Yoshida K, Yoshida N, Nakamura H, Iwaku M, Ozawa H (1996) Immunolocalization of fibronectin during reparative dentinogenesis in human teeth after pulp capping with calcium hydroxide. J Dent Res. 75(8): 15901597.

[12]. Zhang M and Fukuyama H (1999) CGRP immunohistochemistry in wound healing and dentin bridge formation following rat molar pulpotomy. Histochem Cell Biol 112(5): 325-333.

[13]. Osuga N, Matsuda S, Shoumura M, Moriyama K, Nakano K and Kawakami $\mathrm{T}$ (2013) Establishment of experimental periapical inflammatory lesions in mice. J Hard Tissue Bio. 22: 517-520.

[14]. Tsujigiwa H, Nishizaki K, Teshima T, Takeda Y, Yoshinobu J, et al., (2005) The engraftment of transplanted bone marrow-drived cellsinto the olfactory epithelium. Brain Res. 1052(1): 10-15.

[15]. Tomida M, Tsujigiwa H, Nakano K, Muraoka R, Nakamura T, et al., (2013) Promotion of transplanted bone marrow-derived cell migration into the periodontal tissues due to orthodontic mechanical stress. Int J Med Sci. 10(10): 1321-1326.

[16]. Nishizaki K, Yoshinobu J, Tsujigiwa H, Orita Y, Yamada M (2010) The early administration of granulocyte colony-stimulating factor increases the engraftment of transplanted bone marrow-derived cells into the olfactory epithelium damaged by methimazole. Rhinology. 48(2): 228-232.

[17]. Muraoka R, Tsujigiwa H, Naknao K, Katase N, Tamamura R, et al., (2011) Transplanted bone marrow-derived cell migration into periodontal tissues and cell differentiation. J Hard Tissue Biol. 20: 301-306.

[18]. Tsujigiwa H, Katase N, Sathi GA, Buery RR, Hirata Y, et al., (2011) Transplanted bone marrow derived cells differentiated to tooth, bone and connective tissues in mice. J Hard Tissue Biol. 20(2): 147-152.

[19]. Matsuda S, Yokoi Y, Moriyama K, Shoumura M, Osuga N, et al., (2015) Pathological examination of experimentally induced periodontal polyp in mice. J Hard Tissue Biol 24: 397-400.

[20]. Oka Y, Yoshikawa M, Takemura M, Yamamoto K, Toda T (1991) Histological examination on periodontal tissue reaction of rat molar following perforation of the chamber floor. Jpn J Conserv Dent. 34: 1574-1579.

[21]. Imaizumi I, Iwama A, Shibata N, Kumazawa M, Yamasaki M, et al., (1996) Experimental studies on lesion progression following perforation of the furcation area of rat molars. Aichi Gakuin J Dent Sci. 34: 717-723.

[22]. Kudoh J (1989) A study on the treatment of the furcation perforation using germfree rat. Jpn J Conserv Dent. 32: 201-213.

[23]. Ishida T, Tachibana T, Sato K, Sawada M, Watanabe K, et al., (1985) Experimental study of endodontic treatment for furcation perforation. Jpn J Concerv Dent. 28: 1372-1382.

[24]. Tsujigiwa H, Katase N, Sathi GA, Buery RR, Hirata Y, et al., (2011) Transplanted bone marrow derived cells differentiated to tooth, bone and connective tissues in mice. J Hard Tissue Biol. 20(2): 147-152.

[25]. Muraoka R, Tsujigiwa H, Nakano K, Katase N, Tamamura R, et al., (2011) Transplanted bone marrow-derived cell migration into periodontal tissues and cell differentiation. J Hard Tissue Biol. 20(4): 301-306.
[26]. Kaneko K, Matsuda S, Muraoka R, Nakano K, Iwasaki T, et al., (2015) Histological evaluation of periodontal ligament in response to orthodontic mechanical stress in mice. Int J Med Sci. 12(9): 689-694.

[27]. Nakamura C, Hayashi T, Iso K, Nakamura F (1979) A case of periondontal polyp, clinically resembled to pulp polyp. Matsumoto Shigaku (J Matsumoto Dent Univ Sci). 5: 89-93.

[28]. Kramer PR, Kramer SF, Puri J, Grogan D, Guan G (2009) Multipotent adult progenitor cells acquire periodontal ligament characteristics in vivo. Stem Cells Dev. 18(1): 67-75.

[29]. Yamazaki K (2005) Periodontitis and tissue regeneration. Nihon Hotetsu Shika Gakkai Zasshi 49(4): 587-592.

[30]. Nakajima R, Ono M, Hara ES, Oida Y, Shinkawa S, et al., (2014) Mesenchymal stem/progenitor cell isolation from tooth extraction sockets. J Dent Res. 93(11): 1133-1140.

[31]. Okabe M, Ikawa M, Kominami K, Nakanishi T, Nishimune Y (1997) 'Green mice' as a source of ubiquitous green cells. FEBS Lett. 407(3): 313-319.

[32]. Ikawa M, Yamada S, Nakanishi T, Okabe M (1999) Green flourecsent protein (GFP) as a vital maker in mammals. Curr Top Dev Biol. 44: 1-20.

[33]. Zhang J, Chen L, Liu X, Kammertoens T, Blankenstein T, Qin Z (2013) Fibroblast-specific protein 1/S100A4-positive cells prevent carcinoma through collagen production and encapsulation of carcinogens. Cancer Res 73(9): 2770-2781.

[34]. Zeisberg EM, Potenta SE, Sugimoto H, Zeisberg M, Kalluri R (2008) Fibroblasts in kidney fibrosis emerge via endothelial to-mesenchymal transition. J Am Soc Nephrol. 19(12): 2282-2287.

[35]. Kong P, Christia P, Saxena A, Su Y, Frangogiannis NG (2013) Lack of specificity of fibroblast-specific protein 1 in cardiac remodeling and fibrosis. Am J Physiol Heart Circ Physiol. 305(9): 1363-1372.

[36]. Duarte WR, Iimura T, Takenaga K, Ohya K, Ishikawa I, Kasugai S (1999) Extracellular role of S100A4 calcium-binding protein in the periodontal ligament. Biochem Biophys Res Commun. 255(2): 416-420.

[37]. Saito Y, Yoshizawa T, Takizawa F, Ikegame M, Ishibashi O, et al., (2002) A cell line with characteristics of the periodontal ligament fibroblasts is negatively regulated for mineralization and Runx $2 / \mathrm{Cbfa} 1 / \mathrm{Osf} 2$ activity, part of which can be overcome by bone morphogenetic protein-2. J Cell Sci. 115(21): 4191-4200.

[38]. Watanabe T, Nakano K, Shimizu T, Okafuji N, Kurihara S, et al., (2009) Immunohistochemistry of the periodontal ligament fibroblasts in orthodontic tension sides. J Hard Tissue Biol. 18(4): 175-180.

[39]. Sakai K, Nakano K, Matsuda S, Tsujigiwa H, Ochiai T, et al., (2016) Pathological analysis of cell differentiation in Cholesterol Granulomas experimentally induced in mice. Int J Med Sci. 13(3): 220-224.

[40]. Matsuda S, Shoumura M, Osuga N, Tsujigiwa H, Nakano K, et al., (2016) Migration and Differentiation of GFP-transplanted Bone Marrow-derived Cells into Experimentally Induced Periodontal Polyp in Mice. Int J Med Sci. 13(7): 500-506.

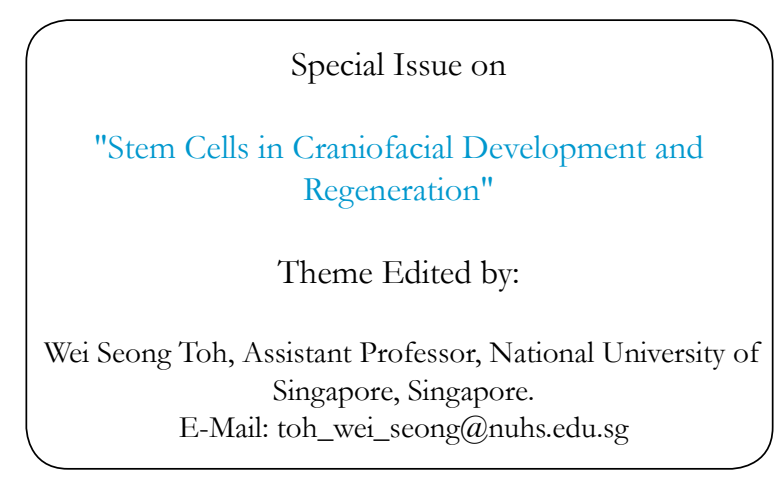

